\title{
Mental Health Outcomes of COVID-19 Quarantine and Social Isolation among MENA Region: A Rapid Review
}

Ms. Shaima Salim Nasser Bani Oraba. BSN ${ }^{1}$, Ms. Azza Zahran Sulaiman Al-Fahdi, BSN ${ }^{1}$, Ms, Asma Saif Salim AlRawahi BSN ${ }^{1}$, Ms. Mabrouka Faraj Abdullah Al-Rawahi BSN ${ }^{1}$, Ms. Sophia Cyril Vincent. MSN ${ }^{2 *}$

${ }^{1}$ Student, College of Nursing, Sultan Qaboos University, Al Khoud, P.O box -66, Postal code-123 Muscat, Sultanate of Oman

${ }^{2}$ Lecturer, College of Nursing, Sultan Qaboos University, Al Khoud, P.O box -66, Postal code-123 Muscat, Sultanate of Oman

$\begin{array}{cl}\text { Article History } & \text { Abstract: Background: The COVID-19 pandemic has caused the most serious health crisis } \\ \text { Received: } 24.02 .2021 & \text { in modern times worldwide, challenging the healthcare systems to contempt conventional } \\ \text { practices to prevent exposure to infection and death. Deception of information from social } \\ \text { media caused a psychological burden due to fear of contamination in the Middle East and } \\ \text { Published: } 15.04 .2021\end{array} \quad \begin{aligned} & \text { North Africa (MENA) region. Method: This rapid review study was conducted to provide } \\ & \text { evidence on the psychological burden in the Middle East North Africa (MENA) regions and } \\ & \text { to prevent the psychological burden during any pandemic situation. Results: Opportunities } \\ & \text { for Mental health promotion are very minimal or still emerging in the MENA region. } \\ & \text { Journal homepage: }\end{aligned} \quad \begin{aligned} & \text { Also, there is a lack of concerted research evidence for mental health impacts during } \\ & \text { previous pandemics like severe acute Respiratory Syndrome (SARS), Ebola, and Middle } \\ & \text { East Respiratory Syndrome (MERS). MENA region is evaded many opportunities to } \\ & \text { advance mental health promotion activities and its preventive measures during quarantine, } \\ & \text { creating the negative effects which are mentioned in the studies as anxiety, stress, fear, sleep } \\ & \text { disturbance, and depression. Conclusion: The psychological impact of COVID 19 has been } \\ & \text { significant among the MENA region population. Many countries in the MENA region have } \\ & \text { an opportunity to quickly strengthen and accord significantly to combat against the } \\ & \text { psychological impact during the pandemic. } \\ & \text { Keywords: Mental Health Outcomes of COVID 19, Social Isolation, MENA Region. }\end{aligned}$

Copyright ( ) 2021 The Author(s): This is an open-access article distributed under the terms of the Creative Commons Attribution 4.0 International License (CC BY-NC 4.0) which permits unrestricted use, distribution, and reproduction in any medium for non-commercial use provided the original author and source are credited.

\section{INTRODUCTION}

The pandemic COVID -19 showed how consistent our world is: No one is safe until everyone is safe. Only solidarity can save lives and overcome the devastating socio-economic impact of the virus. For the past year, the world has been experiencing a global public health pandemic as COVID 19 emerges as a threatening pandemic [1]. COVID -19 a new contagious disease caused by SARS-Cov-2 is a new strain of acute respiratory syndrome. According to the National Health Commission of China, individuals carrying the virus can infect others through both respiratory droplets and direct contact [2]. The severity of the disease is evident from the rapid spread of the virus, its high mortality rate, its new variants, and the recurrence of infection in previously affected individuals. The COVID 19 virus was first identified on December 31, 2019, in the city of Wuhan, China. WHO also reported several cases of pneumonia of unknown cause, which later became known as the new 2019-nCoV. The same patch of the virus was reported in four other countries by January 20, 2020[3].
The WHO declared COVID19 as a pandemic and a public health emergency of global concern on January 30, 2020. By the end of February 2020, the virus had spread to more than 24 countries and the disease was officially designated as COVID -19[4].

The number of positive individuals continues to rise and by May 2020, the virus had spread to all regions of the world. By the end of 2020 and early 2021 , over $87,015,526$ cases and $1,878,581$ deaths had been recorded worldwide. (www.worldometers.info/coronavirus). In the MENA region, Lebanon is the first country to report COVID 19 in February 2020, followed by Algeria, a nation much closer to Europe and in northern Africa that has implemented a full lockdown due to the spread of the virus. According to the Algerian Ministry of Health, Population and Hospital Reform (MSPRH) [5] surveillance cell, the number of confirmed cases exceeded $101 \mathrm{~K}$, which also recorded 2,792 deaths by December 2020. On March 2, the first case of COVID 19 was recorded in Saudi Arabia and since then 36, 3000 confirmed cases 6,278 have been reported by the government of this region to the WHO [6]. Oman is one 
of the regions affected by this pandemic and the first two cases were reported on February 24, 2020 when some citizens returned home after traveling abroad. The Ministry of Health had recently reported a growing number of people testing positive with minimal deaths and various recoveries. Approximately 500 confirmed cases/day indicated by Ministry of Health after mass testing [7].

The first measure taken in the MENA region to prevent the spread of infection is quarantine. Quarantine is one of the oldest and most effective tools for controlling communicable disease outbreaks. Quarantine separates people and restricts their movement, leading to social isolation. Prolonged quarantine, fear of infection, frustration, boredom, inadequate supplies, inadequate information, financial loss, and stigma affect the physical and mental health of the population regardless of age and gender [8]. Not only physical activity but also mental health is affected during quarantine [9], leading to depression, emotional disturbance, stress, low mood, irritability or insomnia, and suicidal ideation, especially in the elderly [10]. A study conducted with pregnant women in the second and third trimesters of pregnancy shows that approximately $35 \%$ of pregnant women self-isolated to avoid exposure to novel coronavirus (COVID -19). In addition, the flood of vague and unreliable information from various sources has caused panic and anxiety among pregnant women, affecting fetal development [11]. One study showed that as a result of COVID19 blocking and online instruction, children and adolescents became less inactive, spent more time with electronics, their sleep patterns were disrupted, and their eating patterns increased, causing them to gain weight. Children experienced isolation from their extended families, peers, teachers and wider communities [12]. In addition, an international study including data from various countries in North and South America, Asia, Europe, and Australia shows that untreated schizophrenia (32.2\%), alcohol disorders $(78.1 \%)$ and major depression $(56.3 \%)$ led to social isolation during the pandemic and in turn accelerated mental health problems and suicidal thoughts [13]. The study also observed that COVID -19 pandemic anxiety, stress, insomnia, depression, and fear of infection were more prevalent among healthcare professionals, people with pre-existing psychiatric disorders, and those living in areas with a high prevalence of COVID -19.

In the Arab countries of the Middle East and North Africa (MENA) region, the total population is 449 million and the health care system in this region is still in transition. A few Arab countries of the MENA region have the privilege, either from the government or private sectors, to meet the challenges of mental health, but it is frequently overlooked as they still consider mental health issues as societal stigma [14].
In MENA region, $75 \%$ of health issues is related to the communicable diseases and injury except in Sudan and Yemen, the lower middle-income countries, instead they account for burden of infectious and parasitic diseases, also account for a significant share of DALYs (Disability-adjusted life years). i.e.loss of life lost due to both premature death due to Cardiovascular disorders (CVDs), mental and behavioral disorder, DM and cancer are health burden in most of the MENA countries[15].

\section{Why is this Review needed?}

During Covid-19 outbreak, national bodies, international bodies and institutions have ordered physical distancing, social isolation and quarantine, to condense the transmission of the virus [16]. These measures are often an unpleasant event for population globally. Parting from society and family, isolation, ambiguity over disease status, and dullness can, create intense physiological and psychological effects [17]. Increased suicidal rate, substantial anger [18] and lawsuits were common following the quarantine guide lines in previous infection epidemics [19]. These strategies also showed other negative impacts on the mental health and wellbeing, such as frustration, loneliness, worries, anxiety [20], affective disorders, and psychoses. Coronavirus-induced distress [21].

In MENA region social mechanism is very minimal and social trust among the wealthier and educated citizens is less appreciated also the religiosity, gender injustice, plays pivot role in all the aspects of life [22]. So, a special care is essential for MENA region population during any pandemic outbreak.

\section{Methodology}

\section{Aim}

We aimed to provide an over view of mental Health Outcomes of COVID 19 Quarantine and social isolation among MENA region population.

\section{Search strategy}

A methodical search was conducted using the data base, Medline, Google Scholar, medRxiv and Pub-med using the English language literature form December 2019 to November 2020.Original research studies and clinical review. Our search terms included "MENA region" or "Mental health" AND "quarantine" or "social isolation" or "loneliness" AND mental health related terms with a focus on the most common mental health problems like depression anxiety and stress.

Rapid review of literature method was conducted as per the National collaborating center for methods \& tools guide book and used PRISMA guidelines for reporting the review. Due to time constraints, one reviewer conducted the search, reviewed full texts and extracted data. Of 786 generated records, we identified 45 full-texts as relevant articles, based on titles and abstracts screening. After examining 
the methodological quality, 11 articles from MENA region were considered as relevant to the review. The most common reason for abstract exclusion was due to not from the MENA region, or not focusing on the Psychological impacts of COVID 19.

\section{Study eligibility criteria}

After screening the tittle and abstract, we included the studies that reported insanity, its associated risk factors, and COVID 19 preventive measures followed, in MENA region. Also, the studies which used standardized and validated tool for psychological measurement of any subgroups (Employees, University students, geriatric). Studies were excluded if they: are other than English, not from MENA region. Psychological impact of pregnant women, if full text not available.

\section{Data charting}

The following were extracted from the eligible studies: Author and year of publication, Country/region of the study conducted, Study design, Sample size, Sample characteristics, used tool of assessment, Prevalence of depression/anxiety/ PTS/stress symptoms, related risk factors, finally preventive measures.

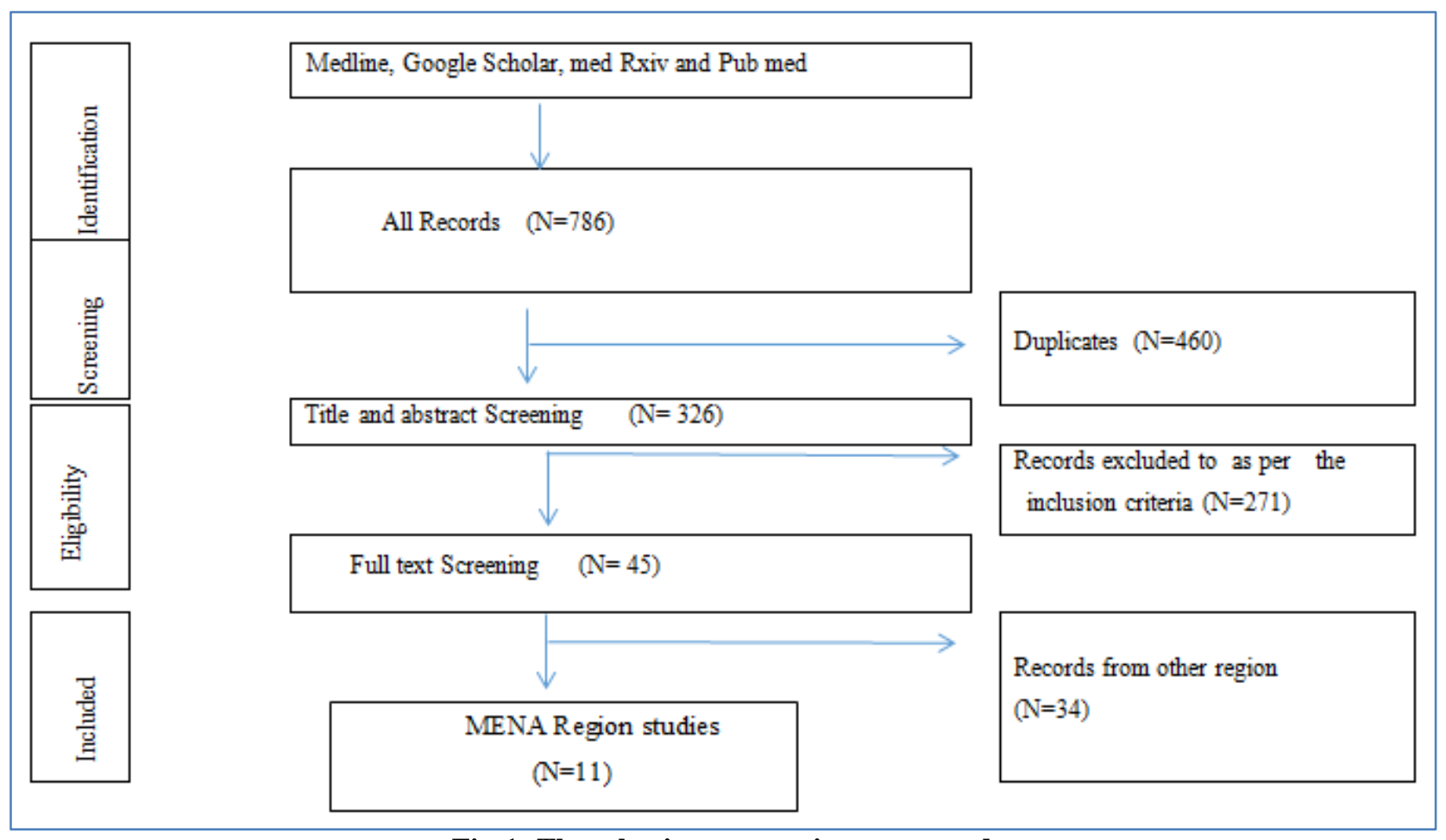

Fig-1: The selection process is represented

\section{RESULTS}

\section{Search results}

Our search identified 786 unique citations, among which 11 studies were included in this review are from MENA regions like: Saudi Arabia -2, Morocco -1, Bahrain -1, Palestine-1, Qatar -1, Egypt -1, UAE-1, Libya-1, Algeria -1 and MENA region -1 .

Fig.1 elaborates the screening process in PRISMA flow diagram and Table-I provide a summary of included studies. Of these 11 articles, Nine (9) studies used cross-sectional research design, one (1) a quantitative transversal study, one (1) clinical review article and interview method.

\section{Study character}

Study samples involved educated, general public of age group above 18-60 years and also the thirteen psychiatrists. Sample selection was mostly through snowball sampling technique. Data collection was on-line either through Twitter, Facebook, Instagram, or google forum, due to the home confinement.

The psychological outcomes of the study chosen are varied across studies. Of the 11 studies included, 10 studies assessed the Psychological impact of MENA region 1 study exceptionally assessed the Correlation between parent's activity and children's mental health during Quarantine.

\section{Measurement tools}

A variety of assessment tools were used to measure impact of social isolation and social distancing on mental well-being. Most commonly used tools was, Depression, Anxiety, and Stress Scale (DASS-21) by 4 studies, other scales are- Event scale Revised (IES-R), Emotional regulation scale (ERQ), Satisfaction with life scale (SWLS). Multidimensional scale of Perceived Social support (MSPSS), Mental Health Continuum 
Scale, GHQ-12 (first part), Hospital Anxiety and Depression Scale (HADS), A Google Form selfadministrated questionnaire for psychological Conditions, semi-structured guide in supplementary tables, global questionnaire, Beck Depression Inventory second edition (BDI-II), perceived Stress Scale (PSS) 10 and mental health score (MCS)

\section{Main findings}

Three main themes emerged from the thematic analysis of the study. 1) Depression and associated risk factors 2) Associated Risk factors anxiety 3) Stress and associated risk factors 4) Mental health Protective factors 5) Preventive measures

\section{Depression and associated risk factors}

Depression signs were measured in 9 of 11 studies [22-32]. The prevalence of severe depression symptoms ranged from 50 to $74 \%$ in which 16.4 to 57.5 $\%$ of population showed moderate to severe depression. In MENA region geriatric group it ranged from 10-46\% [30].

Numerous associated risk factors with depression symptoms were identified in the population MENA region .6 Studies reported that females are most commonly affected by depression, compared to their male counterparts [22, 23, 24, 26, 28, 32]. Participants in the age group $\leq 55$ years showed higher psychological distress in the majority of the selected studies and only one study by El Keshky M et al. [28] noted the reason, as young adults are primary economic supporters and caregivers of the family. Four studies [22, 24, 26, 27] also identified that students and participants with higher education level, working in the medical field, on-site working and has greater prevalence of depressive symptoms. Lack of enjoyment, lack of play, pausing and limitations of many activities led to depression the many students [26].

Further in two studies [22, 30] Parkinson's disease, dementia, pre-existing mental illness, lack of geriatric counseling center, loss of spouse were associated risk factors for depression. Other identified risk factors are duration of quarantine, family violence, lack of sleep ( $<6 \mathrm{hrs})$ especially in college students, a potential risk factor for depression [23, 27].

Mixed results regarding family support and depression were observed in two studies. Family support was positively related to less depression [23], on the other hand one study found an increased prevalence of depression due to constant family support, and lower prevalence of depression due to support from the friends. The predicted reason was the convenience of expressing feelings to a friend rather than a family member [28].

Other risk factors were, poor self-rated health status, known history of mental illness, chronic illness, fear of disease transmission, lack of food supply and living in the urban area, but the reported risk factors for depression are of young adults, female gender, and college students, are more prone to developing mental disorders. Depression is commonly seen in young age groups and in females, as compared to males and older age group.. Other predictive dynamics include duration of lockdown, termination of employment travel to work, presence of a family member or friend with a COVID 19 infection, presence of chronic medical illness, and poor self-rated health.

\section{Symptoms of anxiety and associated risk factors}

Anxiety symptoms and related risk factors were assessed in 7of 11 studies, with an apparent disparity in the prevalence of anxiety symptoms ranging from $13.9 \%$ to $57.5 \%$. Anxiety often co-occurs with depression [33]. Most the depressive and anxiety predictor are identical and include, younger age group ( $\leq 50$ years), lower education level, poor/incorrect selfrated health, isolation, female gender, married couples, quarantine status, fear of being infected, living in containment zone, poor self-rated health status, being lone, low income, isolation status, fear of exposure to infection, unemployment, presence of vulnerable and chronic health conditions at home, history of mental disorders, COVID 19 infected person at home. In addition, frequent exposure of mass media news/ information for more than $2 \mathrm{hrs} / \mathrm{day}$, or watching 5-10 hrs of international TV channels to follow new information was absolutely associated with anxiety symptoms [23, 32].

Further, another study [27] reported that online teaching and postponement of courses was reported to be a risk factor for anxiety in students. Study anxiety was projected as insomnia in $32.5 \%$, unhappiness in $30.5 \%$, feeling constantly strain and poorer ability to cope with problems in $13.6 \%$ of college students. Nevertheless, due to stigma and privacy dilution, majority of the students did not recommend mental health mobile app.

\section{Symptoms of psychological Impact /stress and associated risk factors}

Only one study [22] has reported the prevalence of psychological distress of emerging COVID 19. More than half of the participants $59.7 \%$ had normal scores on the IES-R, but $16.6 \%$ had mild score range, and $17.9 \%$ had severe score range. With respect to stress symptoms, prevalence rates ranged from 13.7 to $55.5 \%$ [22-28]. Despite of using the same measurement scale as i.e., DASS-21 scale. In another study [31] a remarkably different result, on the PSS-10 scale, $63 \%$ of the participants reported normal to mild stress. $35.5 \%$ moderate to severe stress.

\section{Coping strategy}

Only one study [25] examined the coping strategies during quarantine and a positive effect of 
social distancing. A significant indirect effect on both parents' and children's mental health through parenting activities (play) was observed in the study. This implies that parents' coping skills and engagement in activities with the children play a significant role in promoting mental health of both parents and children. However, the study did not mention the type of activity, duration of activity, the resources used for the activity and children's age group as participants.

\section{Protective factors}

In addition to potential risk factors, three studies also reported individual protective factors to combat the pandemic. Dissemination of knowledge on the coronavirus, up-to-date information about disease transmission treatment modalities, and new guidelines were associated with lower risk of anxiety, depression and stress. It was also observed that $88 \%$ of the general population had confidence in local health authorities $[22,26]$ compared to the social media. In addition, precautionary measures such as repeated hand hygiene 10-40 times a day, wearing, masks, following cough etiquette, limitation of movement, reading a book, watching TV even for 12 hours, avoiding social gatherings also anticipated lower psychological stress levels [22, 26, 32]. Individuals with stress coping personality and avoidant attachment styles, reported fewer symptoms of anxiety and stress.

\section{Psycho social and economic impact}

A study by El- Gimati Y et al., [29] reported that the spread of the COVID19 causes psychological and economic problems in all strata of population. Low marriage rate, no social gathering, closure of shopping malls and entertainment halls, boredom due to long duration of home stay, family violence and stress, anxiety and depression were found in the population with prolonged quarantine. The study also showed that the COVID19 affected the behavior of family members, as well as the society at large. This is due to the fear of illness and death.

\section{DISCUSSION}

In early 2020, WHO recommended, measures such as physical distancing, and quarantine, to prevent the further spread of COVID-19, so most of the government declared a partial or full lock down, with the area marked with different zones depending on the severity of effected population. Higher levels of restrictions resulted in loneliness, high level of psychosocial distress (anxiety, depression and stress). In particular, social isolation has been associated with an increased risk of various mental disorders and somatic diseases in general. Therefore, our review soughs to examine the mental health status of the population in the MENA region population and the associated risk factors for mental distress.

For the first time, this Rapid review examined the impact of COVID19 on the mental health of the
MENA population. The eligible studies in this Rapid Review mainly reported on the magnitude of the mental health impact of COVID19 and associated risk factors in the MENA population. In general, the prevalence of psychiatric symptoms was high in the population when compared to the pre-pandemic period. Throughout the study, differences in prevalence rates were found which could be due to different sample size and disproportionality of sex sample, different measurement scales, different socioeconomic status of the country, educational status, and source of COVID 19 information.

This study examined the psychological effects of COVID 19 among the population of the MENA region and found that depression, anxiety and stress accounted for the largest proportion. $46 \%$ of the population exceeded the cutoff score for potential depression, $57 \%$ of the population exceeded the cutoff score for potential anxiety and $75 \%$ for potential stress during the outbreak of COVID 19. These results were congruent with many studies conducted in China, UK and Germany [33-35]. Some demographic variables such as age and gender might play a role in the psychological well-being of individuals. Our study also found that women were more likely to experience mental health problems, especially depression and anxiety, during the pandemic compared to men. This finding is similar to a recent study that showed that men had a lower chance of developing depression, anxiety, loneliness, and stress [38]. However, the studies did not mention the reasons for the gender difference. The biological determinant of vulnerability to psychosocial stress may be due to hormonal and developmental factors in males [36]. A survey in China one month after the outbreak of COVID -19 also reported higher post-traumatic stress symptoms in females [37].

Participants under 55 years of age were more likely to experience psychosomatic symptoms during the pandemic. This could be due to their family responsibilities, financial and emotional support of children or the elderly and caring roles.

Also, a large proportion of individuals under 55years old consist of students and the working population. Students may experience more emotional distress due to closures of university, lower study efficiency with remote online courses, future courses, somatic sadness, low self-esteem and in working population due to deduction of wage, termination from the job, no salary, long term stagnation at home, working at an office with many employees. This is consistent with a study, which assessed the mental status of Chinese college student [38] and Chines employees [39] and noted higher levels of depression, anxiety and stress symptoms during the COVID-19 outbreak. In addition this review noted that working in medical field or family member working in the medical fields as one of the psychological burdens as they deal 
with patient and have more exposure to undiagnosed population. On the other hand, people in a cross sectional study at Singapore reported the high anxiety prevalence among nonmedical health care workers than medical personnel and recommends an early psychological intervention to this vulnerable group [40].

People with a history of chronic illness or medical/ psychiatric illnesses displayed deviation from psychological health; it would be because of postponement of appointment, non-accessibility to medical services and treatment due to partial closure of Hospital units to prevent the spread of disease. Participants, having a family member with chronic medical/ psychiatric condition also showed more stressor, such as social isolation. This is in lane with a study conducted in Singapore [41]. The review also identified that frequent exposure to social media/news relating to COVID-19 as a cause of depression, anxiety and stress symptoms and in a study also mentioned that $80 \%$ of the population confidence in local health authorities information which was congruent with a cohort study of Singapore which reported that $99.1 \%$ of population trusted the COVID 19 related information from official government sources [42]. It may be due to potential fake news about the mode of transmission, lack of accurate information with the impulsive situation and a lot of indecision about the novel coronavirus; constant watching of news also leads to fear and anxious feelings [43]. In addition, a large percentage of people under the age of 55 consist of students and the working population. Students may experience more emotional stress due to university closures, lower study efficiency with online distance learning courses, future courses, somatic sadness, low self-esteem and in the working population due to wage deduction, job termination, no salary, long-term stagnation at home, working in an office with many coworkers. This is consistent with a study that examined the mental status of Chinese college students [38] and Chinese white-collar workers [39] and found higher levels of depression, anxiety, and stress symptoms during the outbreak COVID -19. In addition, this review found that people who work in the medical field or family members who work in the medical field are one of the psychological burdens because they deal with patients and have more contact with undiagnosed people. On the other hand, people in a cross-sectional study in Singapore reported the high prevalence of anxiety among non-medical health workers compared to medical workers and recommend early psychological intervention for this vulnerable group [40]. People with a history of chronic illness or medical/psychiatric conditions showed a deviation from psychological health, whether due to rescheduling of appointments, inaccessibility of medical services and treatments due to partial closure of hospital units to prevent the spread of illness. Participants who have a family member with a chronic medical/psychiatric condition also showed more stressors, such as social isolation. This is in line with a study conducted in Singapore [41]. The review paper also noted that frequent exposure to social media/news related to COVID -19 is a cause of depression, anxiety and stress symptoms and a study also mentioned that $80 \%$ of the population trust information from local health authorities, which is in line with a cohort study from Singapore which reported that $99.1 \%$ of the population trust COVID 19 related information from official government sources [42]. It may be due to possible fake news about the mode of transmission, lack of accurate information with the impulsive situation and much indecision about the novel coronavirus; constant watching of news also leads to fear and anxious feelings [43].

Individual motivational activity also relieves their mental stress. For example, engaging with their children through play or shared activities has been shown to be effective in preventing symptoms of depression or stress. The results also showed that parental activities with children were significantly positively correlated with parents' coping strategies. This means that parents who develop and implement activities with children are more likely to have coping strategies that enable them to deal with stressful circumstances. A study in the Netherlands also found a positive relationship between parent-child activities and children's mental health. The Positive Mental health of both children and parents may be attributable [44]. In one study, university students recommended a mobile app for coping with mental health issues, and a review paper identified a stress management app available in the iOS app store that provides critical guidance and health-related self-management apps for the future [45].

The strength of our work is, this is the first rapid review that summarizes the existing literature, relevant to the mental health of the MENA population during the COVID19 outbreak and highlights its potential risk factors to provide suggestions for addressing the mental health crisis among the population of the MENA region, although this review has certain limitations. The findings of the studies were qualitative and narrative and due to the lack of time, the reviews were not peer reviewed. In addition, all studies were conducted virtually and the questionnaire was only completed by participants who had a basic knowledge of using the internet and the required application. Due to the lack of studies conducted in each of the MENA countries, it was difficult to conduct a rapid review covering all MENA regions. In addition, the number of articles used in this review was smaller; this affects the generalizability of the findings. Crucially, the mental health problems caused by social isolation and disengagement, and ways to overcome them, need to be comprehensively highlighted

\section{CONCLUSION}


This rapid review surveyed the psychological status of the MENA region population during the COVID19 pandemic and emphasized the related risk factors. An increased incidence of adverse symptoms of psychiatric was stated in most studies. The COVID-19 pandemic represents a unique threat to mental health globally irrespective of the socioeconomic status. Hence, priority needs to be given to the prevention of mental disorders (e.g. major depressive disorder, anxiety, and stress).An integration of government policy that focus on viral risk mitigation and mental health strategies during pandemic is urgently needed for MENA region.

Authorship contribution statement -Ms. Sophia Cyril contributed to the overall design, article selection, review, and manuscript preparation. Ms. Shima Shaima Salim Nasser and Ms. Azza Zahran Sulaiman Ms. Asma Saif, and Ms. Mabrouka Faraj contributed to the study in preparation of the study matrix, result from analysis, discussion. Ms. Shiama contributed to reviewing the article, Ms. Sophia in editing, and submission. Declaration of Competing Interest None. It is a nonfunded study

\section{REFERENCES}

1. Banerjee, D., \& Rai, M. (2020). Social isolation in Covid-19: The impact of loneliness.

2. Klompas, M., Baker, M. A., \& Rhee, C. (2020). Airborne transmission of SARS-CoV-2: theoretical considerations and available evidence. Jama.

3. Mohanty, S. K., Satapathy, A., Naidu, M. M., Mukhopadhyay, S., Sharma, S., Barton, L. M., \& Parwani, A. V. (2020). Severe acute respiratory syndrome coronavirus-2 (SARS-CoV-2) and coronavirus disease 19 (COVID-19)-anatomic pathology perspective on current knowledge. Diagnostic pathology, 15(1), 1-17.

4. World Health Organization (WHO) Emergency Committee. Statement on the second meeting of the International Health Regulations. (2005). Emergency Committee regarding the outbreak of novel coronavirus (2019-nCoV). Geneva: WHO; 30 January 2020. Available from: https://www.who.int/ news-room/detail/30-012020-statement-on-the-secondmeeting-of-theinternational-health-regulations-(2005)-emergencycommittee-regarding-the-outbreak-ofnovelcoronavirus-(2019-ncov)

5. TALEB, S. M., \& BOUSSAKTA, M. A. (2020). The first thousands of cases of coronavirus disease 2019 (COVID-19) in Algeria: some risk factors. medRxiv.

6. Alhumaid, J., Ali, S., \& Farooq, I. (2020). The psychological effects of the COVID-19 pandemic and coping with them in Saudi Arabia. Psychological Trauma: Theory, Research, Practice, and Policy, 12(5), 505-507.

7. Alshekaili, M., Hassan, W., Said, N. A., Sulaimani, F. A., Jayapal, S. K., Al-Mawali, A., Al-Adawi, S.
(2020). Factors associated with mental health outcomes across healthcare settings in Oman during COVID-19: Frontline versus non-frontline healthcare workers. BMJ Open, 10(10).

8. Brooks, S. K., Webster, R. K., Smith, L. E., Woodland, L., Wessely, S., Greenberg, N., \&amp; Rubin, G. J. (2020). The Psychological Impact of Quarantine and How to Reduce It: Rapid Review of the Evidence. SSRN Electronic Journal.

9. Benke, C., Autenrieth, L. K., Asselmann, E., \&amp; Pané-Farré, C. A. (2020). Lockdown, quarantine measures, and social distancing: Associations with depression, anxiety and distress at the beginning of the COVID-19 pandemic among adults from Germany. Psychiatry Research, $293,113462$.

10. Sepúlveda-Loyola, W., Rodríguez-Sánchez, I., Pérez-Rodríguez, P., Ganz, F., Torralba, R., Oliveira, D. V., \& Rodríguez-Mañas, L. (2020). Impact of social isolation due to COVID-19 on health in older people: Mental and physical effects and recommendations. The journal of nutrition, health \& aging, 1-10.

11. Begum, M. R., Ehsan, N., Ehsan, M., \&amp; Sharif, A. B. (2020). Pregnancy and Childbirth in COVID-19Positive/Probable and Suspected Patients: A Comprehensive Review. Journal of Bangladesh College of Physicians and Surgeons, 91-108.

12. Dib, S., Rougeaux, E., Vázquez-Vázquez, A., Wells, J. C., \& Fewtrell, M. (2020). The impact of the COVID-19 lockdown on maternal mental health and coping in the UK: Data from the COVID-19 New Mum Study. medRxiv

13. Loades, M. E., Chatburn, E., Higson-Sweeney, N., Reynolds, S., Shafran, R., Brigden, A. . . Crawley, E. (2020). Rapid Systematic Review: The Impact of Social Isolation and Loneliness on the Mental Health of Children and Adolescents in the Context of COVID-19. Journal of the American Academy of Child \&amp; Adolescent Psychiatry, 59(11).

14. Sher, L. (2020). The impact of the COVID-19 pandemic on suicide rates. QJM: An International Journal of Medicine, 113(10), 707-712.

15. Joshan, S., \& Maertens, S. (2020). Low cost carriers in the Middle East and North Africa (MENA) region: Emergence and barriers to development. Journal of Transport Geography, 87, 102799.

16. Barbisch, D., Koenig, K. L., \& Shih, F. Y. (2015). Is there a case for quarantine? Perspectives from SARS to Ebola. Disaster medicine and public health preparedness, 9(5), 547-553.

17. Rubin, G. J., \& Wessely, S. (2020). The psychological effects of quarantining a city. Bmj, 368.

18. Asbu, E. Z., Masri, M. D., \& Kaissi, A. (2017). Health status and health systems financing in the MENA region: roadmap to universal health 
coverage. Global health research and policy, 2(1), 1-13.

19. Soklaridis, S., Lin, E., Lalani, Y., Rodak, T., \& Sockalingam, S. (2020). Mental health interventions and supports during COVID- 19 and other medical pandemics: A rapid systematic review of the evidence. General hospital psychiatry, 66, 133-146. https://doi.org/10.1016/j.genhosppsych.2020.08.00 7

20. Williams, S. N., Armitage, C. J., Tampe, T., \&amp; Dienes, K. (2020). Public perceptions and experiences of social distancing and social isolation during the COVID-19 pandemic: A UK-based focus group study. Med Rxiv.

21. Kim, H. H. S., \& amp; Jung, J. H. (2020). Social Isolation and Psychological Distress during the COVID-19 Pandemic: A Cross-National Analysis. The Gerontologist.

22. Alkhamees, A. A., Alrashed, S. A., Alzunaydi, A. A., Almohimeed, A. S., \& Aljohani, M. S. (2020). The psychological impact of COVID-19 pandemic on the general population of Saudi Arabia. Comprehensive psychiatry, 102, 152192.

23. 23. Arafa, A., Mohamed, A., Saleh, L., \& Senosy, S. (2020). Psychological Impacts of the COVID-19 Pandemic on the Public in Egypt. Community mental health journal, 1-6.

24. Alsalman, A., Mubarak, H., Aljabal, M., Abdulnabi, M., Ishaq, A., Yusuf, A., \& Jahrami, H. (2020). The Psychological Impact of COVID-19 Pandemic on the Population of Bahrain.

25. Abdelrahman, M., Al-Adwan, D., \& Hasan, Y. (2020). Impact of social distancing on the mental health of parents and children in Qatar.

26. Al Zabadi, H., Haj-Yahya, M., Yaseen, N., \& Alhroub, T. (2020). The role of Coronavirus Disease (COVID-19) on anxiety and stress among the Palestinian general population: A cross sectional study.

27. Drissi, N., Alhmoudi, A., Nuaimi, H. A., Alkhyeli, M., Alsalami, S., \&amp; Ouhbi, S. (2020). Investigating the Impact of COVID-19 Lockdown on the Psychological Health of University Students and Their Attitudes Toward Mobile Mental Health Solutions: Two-Part Questionnaire Study. JMIR Formative Research, 4(10). doi:10.2196/19876

28. El Keshky, M. E. S., Basyouni, S., \& Al Sabban, A. (2020). The psychological and social impacts on personal stress for residents quarantined for COVID-19 in Saudi Arabia.

29. Elgimati, Y., Alrasheed, A., \& Bashir, A. M. (2020). Effect of a COVID-19 on Social, Psychological, Economic and Health Conditions in Libya. Journal of Applied Science, Engineering, Technology, and Education, 3(2), 160-170.

30. El Hayek, S., Cheaito, M. A., Nofal, M., Abdelrahman, D., Adra, A., Al Shamli, S.\& Emberish, A.M. (2020). Geriatric mental health and COVID-19: An eye-opener to the situation of the Arab countries in the Middle East and North Africa Region.

31. Maliki, I., Elmsellem, H., Hafez, B., EL Moussaoui, A., Reda Kachmar, M., \& Ouahbi, A. (2020). The psychological properties of the Arabic BDI-II and the psychological state of the general Moroccan population during the mandatory quarantine due to the COVID-19 pandemic.

32. Madani, A., Boutebal, S. E., \& Bryant, C. R. (2020). The Psychological Impact of Confinement Linked to the Coronavirus Epidemic COVID-19 in Algeria. International Journal of Environmental Research and Public Health, 17(10), 3604. doi:10.3390/ijerph17103604

33. Zhu, S., Wu, Y., Zhu, C. Y., Hong, W. C., Yu, Z. X., Chen, Z. K., ... \& Wang, Y. G. (2020). The immediate mental health impacts of the COVID-19 pandemic among people with or without quarantine managements. Brain, behavior, and immunity.

34. Pierce, M., Hope, H., Ford, T., Hatch, S., Hotopf, M., John, A., \& Abel, K. M. (2020). Mental health before and during the COVID-19 pandemic: a longitudinal probability sample survey of the UK population. The Lancet Psychiatry, 7(10), 883-892.

35. Benke, C., Autenrieth, L. K., Asselmann, E.,\& amp; Pané-Farré, C. A. (2020). Lockdown, quarantine measures, and social distancing: Associations with depression, anxiety and distress at the beginning of the COVID-19 pandemic among adults from Germany. Psychiatry Research, 293, 113462.

36. Wang, J., Korczykowski, M., Rao, H., Fan, Y., Pluta, J., Gur, R. C. ... \& Detre, J. A. (2007). Gender difference in neural response to psychological stress. Social cognitive and affective neuroscience, 2(3), 227-239.

37. Liu, N., Zhang, F., Wei, C., Jia, Y., Shang, Z., Sun, L., \& Liu, W. (2020). Prevalence and predictors of PTSS during COVID-19 outbreak in China hardest-hit areas: Gender differences matter. Psychiatry research, 287, 112921.

38. Wang, C., \& Zhao, H. (2020). The impact of COVID-19 on anxiety in Chinese university students. Frontiers in psychology, 11, 1168.

39. Shi, L., Lu, Z. A., Que, J. Y., Huang, X. L., Liu, L., Ran, M. S., ... \& Lu, L. (2020). Prevalence of and risk factors associated with mental health symptoms among the general population in China during the coronavirus disease 2019 pandemic. JAMA network open,3(7), e2014053e2014053.

40. Tan, B. Y., Chew, N. W., Lee, G. K., Jing, M., Goh, Y., Yeo, L. L.,\& Sharma, V. K. (2020). Psychological impact of the COVID-19 pandemic on health care workers in Singapore. Annals of internal medicine, 173(4), 317-320.

41. Ho, C. S., Chee, C. Y., \& Ho, R. C. (2020). Mental health strategies to combat the psychological impact of COVID-19 beyond paranoia and panic. Ann Acad Med Singapore, 49(1), 1-3. 
42. Lim, V. W., Lim, R. L., Tan, Y. R., Soh, A. S., Tan, M. X., Othman, N. B., ... \& Chena, M. I. (2021). Government trust, perceptions of COVID19 and behaviour change: cohort surveys, Singapore. Bull World Health Organ, 99, 92-101.

43. Sharma, P., Gupta, S., Kushwaha, P., \& Shekhawat, K. (2020). Impact of mass media on quality of life during COVID-19 pandemic among Indian population. Int J Sci Healthc Res, 5, 260-7.
44. Roeters, A., \& van Houdt, K. (2019). Parent-Child Activities Paid Work Interference and Child Mental Health. Family Relations, 68(2), 232-245.

45. Coulon, S. M., Monroe, C. M., \& West, D. S. (2016). A systematic, multi-domain review of mobile smartphone apps for evidence-based stress management. American journal of preventive medicine, 51(1), 95-105.

\begin{tabular}{|c|c|c|c|c|c|c|c|c|}
\hline & 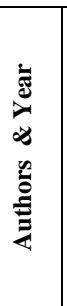 & \begin{tabular}{|l} 
\\
$\frac{\pi}{2}$
\end{tabular} & 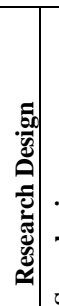 & 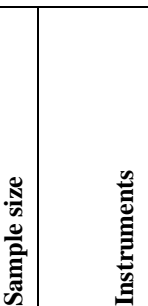 & 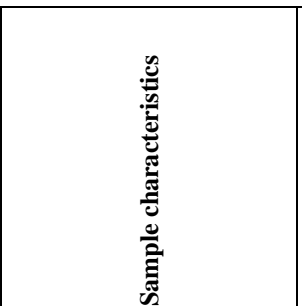 & 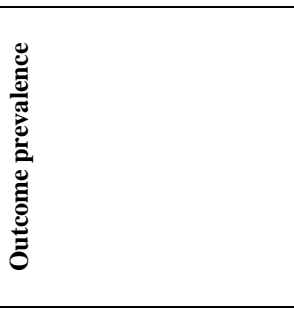 & 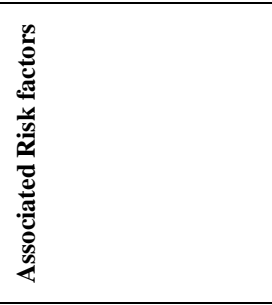 & 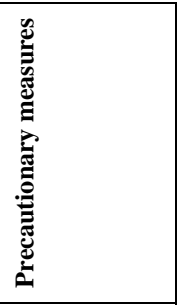 \\
\hline ส & 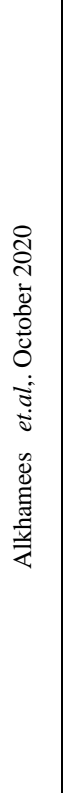 & 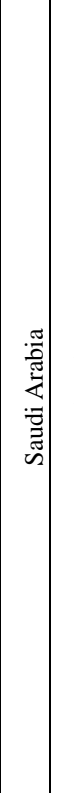 & 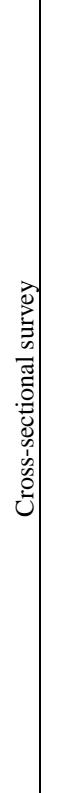 & 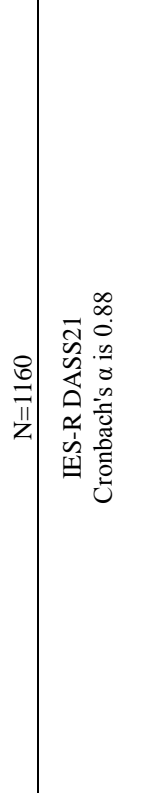 & 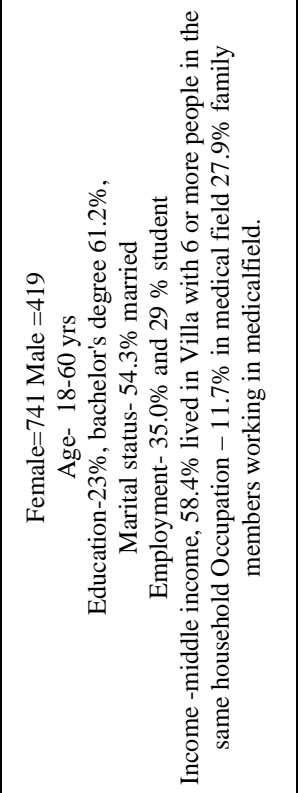 & 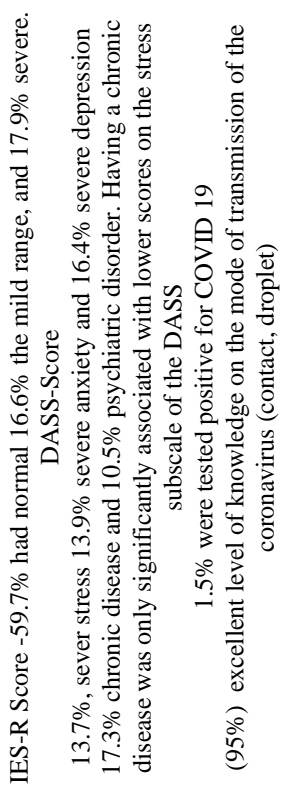 & 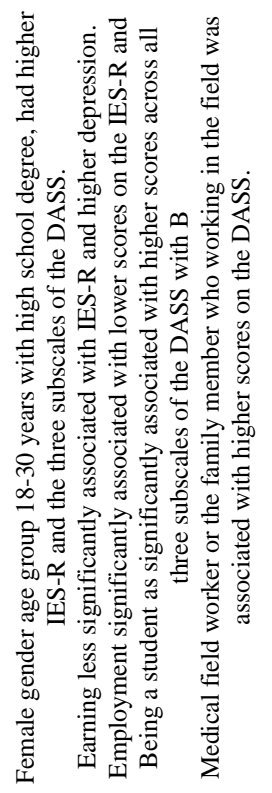 & 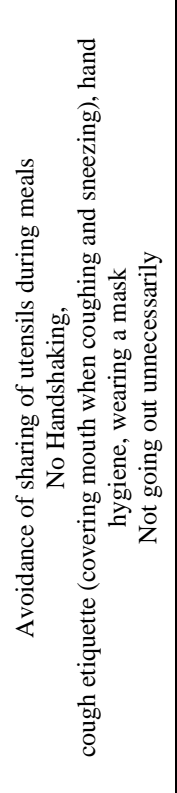 \\
\hline & 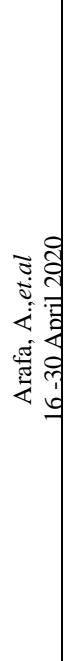 & 咅 & 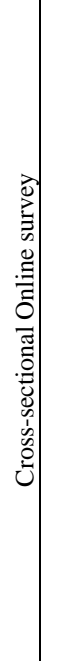 & 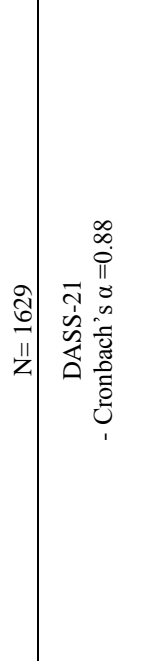 & 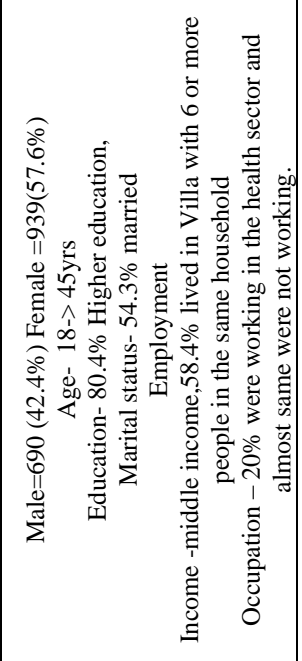 & 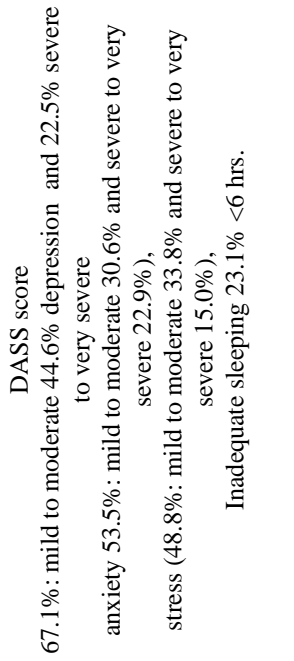 & 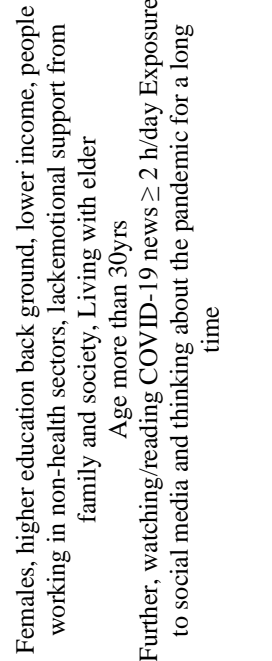 & \\
\hline
\end{tabular}




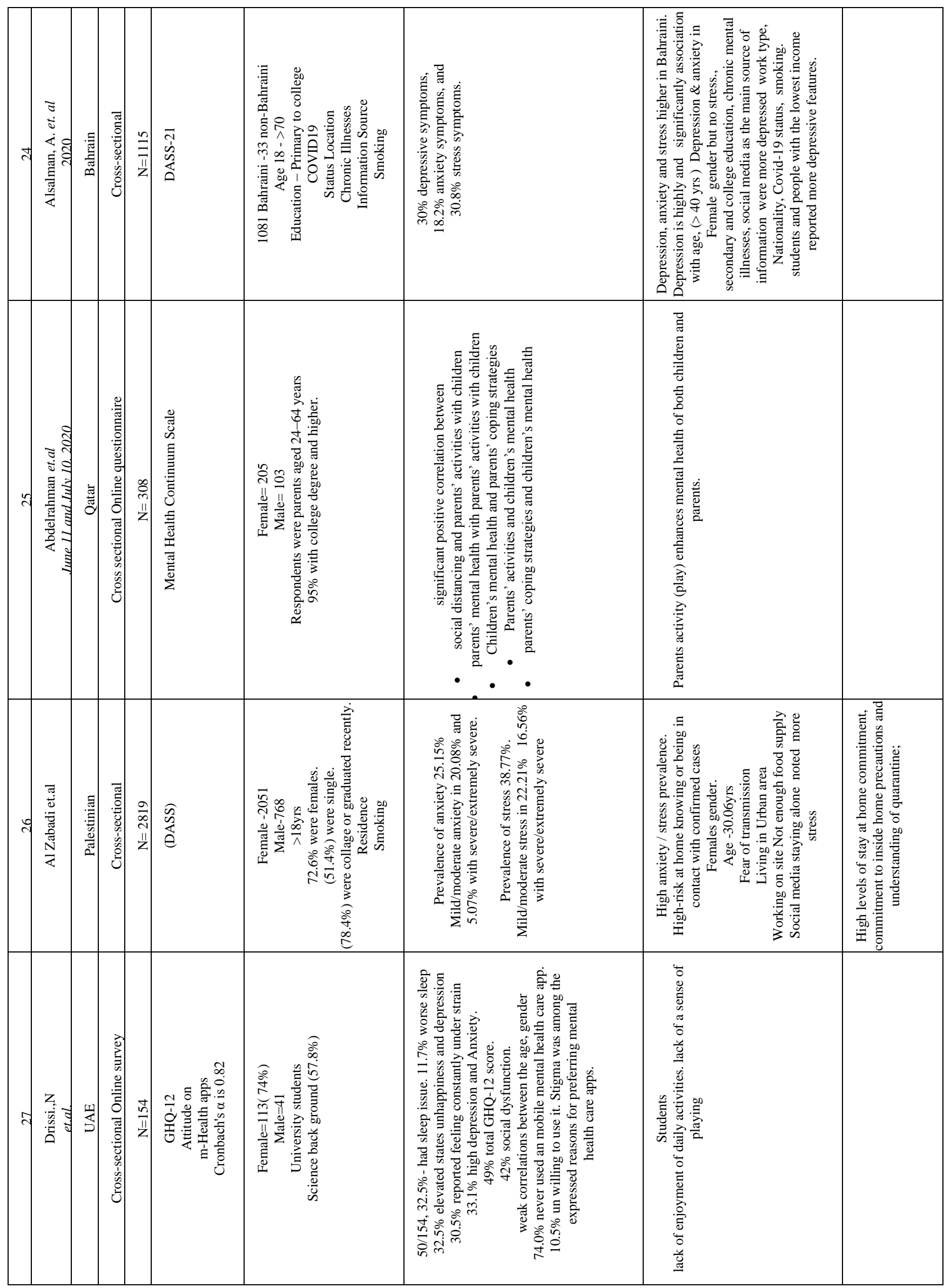




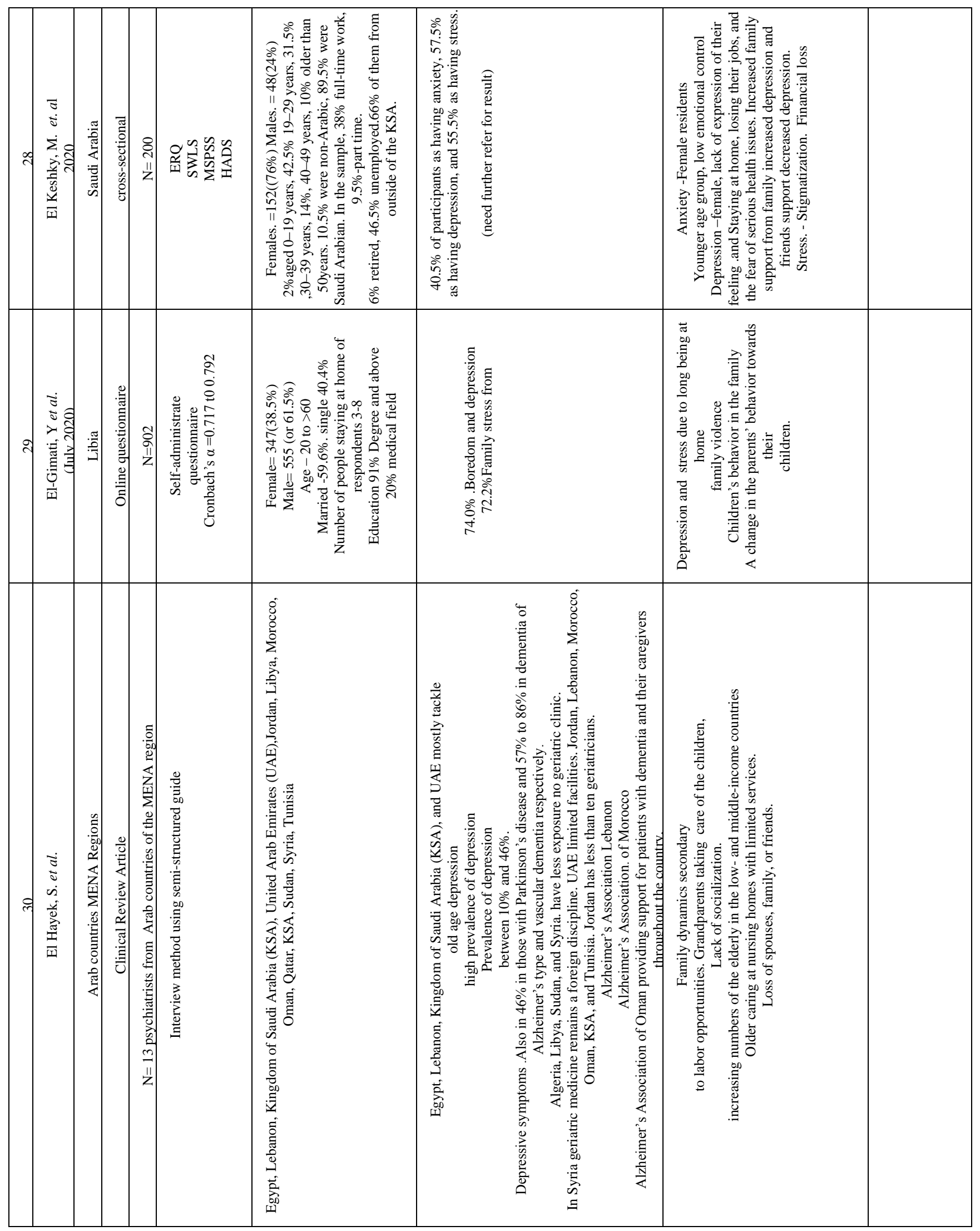




\begin{tabular}{|c|c|c|c|c|c|c|c|c|c|}
\hline$\Rightarrow$ & 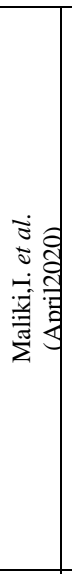 & $\begin{array}{l}0 \\
8 \\
\dot{0} \\
\Sigma\end{array}$ & 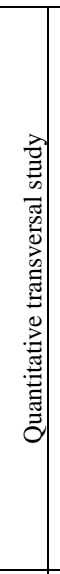 & 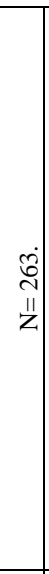 & 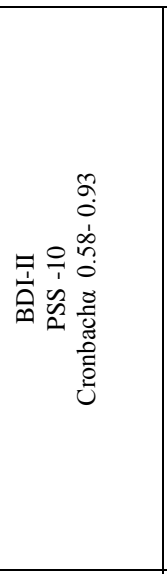 & 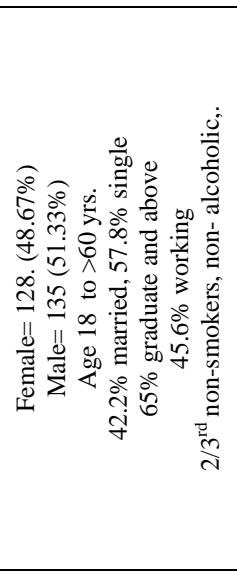 & 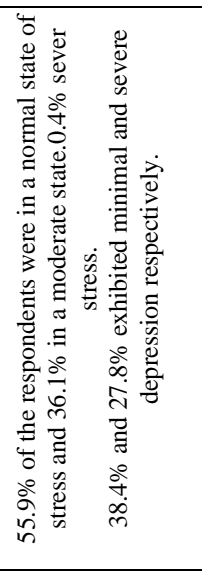 & 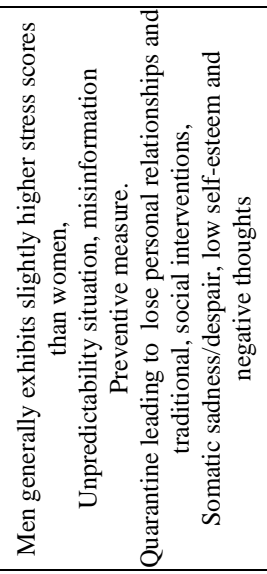 & \\
\hline A & 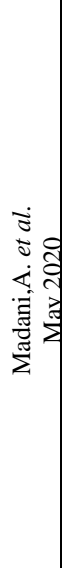 & 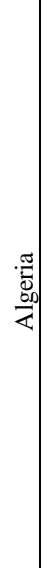 & 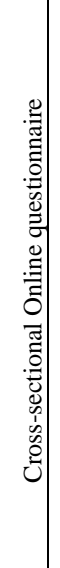 & $\begin{array}{c} \\
\infty \\
0 \\
\\
z \\
z\end{array}$ & 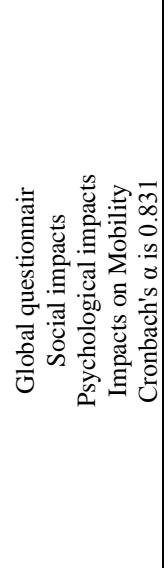 & 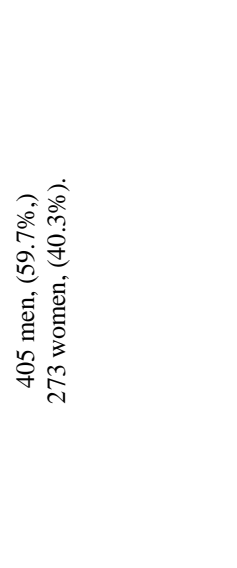 & 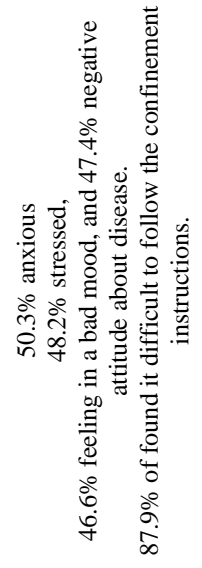 & 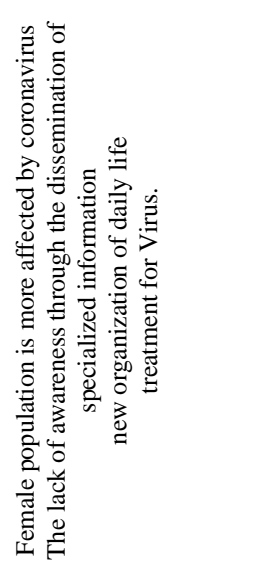 & 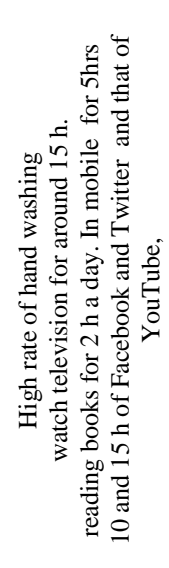 \\
\hline
\end{tabular}

Depression, Anxiety, and Stress Scale (DASS-21) Event scale Revised (IES-R), Emotional regulation scale (ERQ), Satisfaction with life scale (SWLS). Multidimensional scale of Perceived Social support (MSPSS), Hospital Anxiety and Depression Scale (HADS), Beck Depression Inventory second edition (BDI-II), Perceived Stress Scale (PSS) -10 mental health score (MCS) Short form Health Survey (SF-12).

Cite This Article: Shaima Salim Nasser Bani Oraba (2021). Mental Health Outcomes of COVID-19 Quarantine and Social Isolation among MENA Region: A Rapid Review. EAS J Nurs Midwifery, 3(2), 82-93. 